\title{
miR-181a-5p, an inducer of Wnt-signaling, facilitates cell proliferation in acute lymphoblastic leukemia
}

\author{
XIAOMING LYU ${ }^{1}$, JINBANG LI $^{2}$, XI YUN $^{3}$, RUI HUANG $^{4}$, XUBIN DENG $^{5}$, \\ YINGPING WANG $^{6}$, YOUMING $\mathrm{CHEN}^{1}$ and GANG XIAO ${ }^{1}$
}

\begin{abstract}
${ }^{1}$ Department of Laboratory Medicine, The Third Affiliated Hospital of Southern Medical University, Guangzhou, Guangdong; ${ }^{2}$ Department of Pathology, Qingyuan People's Hospital, Jinan University, Qingyuan, Guangdong; ${ }^{3}$ Department of Clinical Laboratory, The Third Affiliated Hospital of Sun Yet-sen University; ${ }^{4}$ Department of Hematology,

Zhujiang Hospital, Southern Medical University; ${ }^{5}$ Department of Medical Oncology, Affiliated Cancer Hospital of Guangzhou Medical University, Guangzhou, Guangdong; ${ }^{6}$ Department of Biochemistry and Molecular Cell Biology, Shanghai Key Laboratory for Tumor Microenvironment and Inflammation, Institute of Medical Sciences, Shanghai Jiao Tong University School of Medicine, Shanghai, P.R. China
\end{abstract}

Received June 13, 2016; Accepted August 22, 2016

DOI: $10.3892 /$ or.2017.5425

\begin{abstract}
Uncontrolled Wnt signaling causes leukemia. Inactivation of Wnt antagonists could play an important role in leukemia progression by activating the $\mathrm{Wnt} / \beta$-catenin pathway. Wnt inhibitory factor-1 (WIF1) is one of the important Wnt antagonists. Few miRNAs have been reported to directly target this gene in hematopoiesis. Here, we observed that miR-181a-5p expression was markedly overexpressed in several leukemia cell lines and acute lymphoblastic leukemia (ALL) samples compared with that noted in normal peripheral blood mononuclear cells. MTT assays, soft agar colony formation assays and flow cytometry analysis collectively showed that ectopic expression of miR-181a-5p induced ALL cell growth and proliferation. Furthermore, a mechanistic study disclosed that miR-181a-5p directly downregulated WIF1 expression by binding to its 3'-UTR, and further activated Wnt $/ \beta$-catenin signaling. These findings provide a novel mechanistic insight into the role of miR-181a-5p in ALL cell growth and proliferation and implicate miR-181a-5p as an attractive candidate for ALL therapy.
\end{abstract}

\section{Introduction}

Acute lymphoblastic leukemia (ALL) is a malignant disorder of lymphoid progenitor cells. The steady progress in the

Correspondence to: Youming Chen or Dr Gang Xiao, Department of Laboratory Medicine, The Third Affiliated Hospital of Southern Medical University, 183 East Zhongshan Road, Guangzhou, Guangdong 510630, P.R. China

E-mail: cym38432@163.com

E-mail: xiaogang2993@yeah.net

Key words: acute lymphoblastic leukemia, miR-181a-5p, Wnt inhibitory factor- $1, \mathrm{Wnt} / \beta$-catenin, cell proliferation development of effective treatments has led to a cure rate of more than $80 \%$ in children with ALL, most of whom will lead healthy productive lives as long-term cancer survivors $(1,2)$. Yet, studies are underway to ascertain the precise events that take place in the genesis of ALL, to enhance the clinical application of known risk factors and anti-leukemic agents, and to identify treatment regimens that may boost the generally low cure rates in adults and subgroups of children with high-risk leukemia (3-5). Approaches aimed at blocking associated miRNAs may serve as effective therapeutic strategies for treating ALL patients and may be valuable biomarkers for diagnosis and treatment.

Dysregulated Wnt signaling has been reported as a hallmark of particular types of solid tumors (6-9). Several studies have also indicated that Wnt signal transduction plays a key role in certain stages of lymphocyte development and the self-renewal of hematopoietic stem cells (10-16), suggesting its dysregulation as a mechanism underlying lymphoid leukemogenesis. Recent studies concerning acute or chronic lymphoid leukemias have provided further evidence for the role of Wnt signaling in malignant hematopoiesis $(10-12,17,18)$.

The deficiency of Wnt antagonists can contribute to activation of the Wnt pathway resulting in carcinogenesis via deregulation of cell proliferation and differentiation. Numerous studies have shown that impaired regulation of Wnt antagonists such as Wnt inhibitory factor-1 (WIF1), sFRP, HDPR 1 and DKK3 by promoter hypermethylation is present in several human malignancies including ALL (17-22). However, it is noteworthy that the frequency of methylation of WIF1 is less than $30 \%$ in ALL (20), which implies that there may be additional regulatory mechanisms responsible for downregulating WIF1 expression.

miRNAs are short (19-25 nucleotides) RNA molecules that can modulate the expression of a wide range of target genes by pairing homologous sequences within the 3'-UTR of mRNAs, thus impairing their translation or promoting RNA degradation $(23,24)$ in multiple diseases including cancers. 
miR-181a has been proposed to play multi-roles in neoplasia and progression. miR-181a-5p is an oncogenic miRNA found to be deregulated in multiple types of tumors [e.g., breast cancer (25-27), osteosarcoma (28), colorectal carcinoma (29), gastric cancer (30), salivary adenoid cystic carcinoma (31) and lung cancer (32)]. In contrast, miR-181a was downregulated in many other tumors and thus served as a tumor-suppressor gene $(33,34)$. In previous studies of hematologic malignancies, miR-181a was found to be upregulated in acute myeloid leukemia (35) and myelodysplastic syndromes (36), but downregulated in multiple myeloma (37) and chronic lymphocyte leukemia (38).

In this study, we demonstrated that upregulation of miR-181a-5p directly targets WIF1 in ALL cells, suggesting that miR-181a-5p-mediated Wnt-signaling activation may be implicated in the pathogenesis of ALL.

\section{Materials and methods}

Cell lines and clinical samples. Two ALL-derived cell lines (Jurkat and MOLT-4) and other hematopoietic tumor cell lineages (HL60, NB4 and KG-1) were obtained from the Cancer Research Institute, Southern Medical University, Guangzhou, China. Cells were cultured at $37^{\circ} \mathrm{C}$ under $5 \% \mathrm{CO}_{2}$ in humidified air in RPMI-1640 medium (HyClone, Logan, UT, USA) supplemented with $10 \%$ fetal bovine serum (Gibco, Grand Island, NY, USA). Thirty-seven primary ALL patients and 28 ALL-complete remission bone marrow samples were collected from The Third Affiliated Hospital and Zhujiang Hospital, Southern Medical University. Twenty-three samples of normal peripheral blood mononuclear cells (PBMCs) were obtained from Guangzhou Blood Center.

The study was approved by the Human Ethics Committee at The Third Affiliated Hospital of Southern Medical University. Written informed consent was obtained from all participants.

Extraction of total RNA and quantitative RT-PCR ( $q R T-P C R)$. Total RNA was extracted from tissues and cell lines with TRIzol (Invitrogen, Carlsbad, CA, USA) according to the user manual. For mRNA expression analysis, $1 \mu \mathrm{g}$ of total RNA was used for RT using PrimerScript ${ }^{\mathrm{TM}}$ RT reagent kit following the manufacturer's instructions (lot. no. BK3001), and real-time PCR was performed using SYBR ${ }^{\circledR}$ Premix Ex Taq ${ }^{\mathrm{TM}}$ Real-Time PCR kit (code no. DRR041A) (both from Takara Bio) on an Mx3005P Stratagene. All data were normalized to GAPDH expression and further normalized to the negative control unless otherwise indicated. Primer sequences for WIF-1 (forward, 5'-TCTCCAAACACCTCAAAATGCT-3' and reverse, 5'-GACACTCGCAGATGCGTCT-3') and $G A P D H$ (forward, 5'-CCATGAGAAGTATGACAACAGCC-3' and reverse, 5'-GGGTGCTAAGCAGTTGGTG-3') were directly acquired from PrimerBank (http://pga.mgh.harvard. edu/primerbank/). For miRNA expression analysis, mature miRNAs were reverse-transcribed, and real-time PCR was performed using All-in-One ${ }^{\mathrm{TM}}$ miRNA qRT-PCR detection kit following the manufacturer's instructions (cat. no. AOMD-Q020; GeneCopoeia, Inc., Rockville, MD, USA). All data were normalized to $U 6$ expression. The fold changes were calculated by relative quantification $\left(2^{-\Delta \Delta \mathrm{Ct}}\right)$. qRT-PCR was conducted for each sample in triplicate.
MTT assay. MOLT-4 $\left(5 \times 10^{3}\right)$ and Jurkat cells $\left(3 \times 10^{3}\right)$ were plated onto 96-well plates (NEST Biotechnology, Wuxi, China) respectively in $100 \mu \mathrm{l}$ growth medium and allowed to adhere overnight. The cells were then transfected with $50 \mathrm{nM}$ of miRNA mimics (GenePharma, Shanghai, China) or siRNA (Santa Cruz Biotechnology, Inc., Santa Cruz, CA, USA) and inhibitor (GenePharma), respectively. At different time-points (24,48 and $72 \mathrm{~h})$, the culture medium was removed and replaced with culture medium containing $10 \mu \mathrm{l}$ of sterile 3-(4,5-dimethyl2-thiazolyl)-2,5-diphenyl-2H-tetrazolium bromide (MTT) dye $(5 \mathrm{mg} / \mathrm{ml})$. After incubation at $37^{\circ} \mathrm{C}$ for $4 \mathrm{~h}$, the MTT solution was removed, and $150 \mu 1$ dimethyl sulfoxide (DMSO) was added to dissolve the formazan crystals. Spectrometric absorbance at $490 \mathrm{~nm}$ was measured using a BioTek ELx800 microplate photometer (BioTek ELx800, SN211805; BioTek, Winooski, VT, USA).

Anchorage-independent growth assay. Cells were blowed gently, and $1 \times 10^{5}$ cells were resuspended in $2 \mathrm{ml}$ complete medium plus $0.3 \%$ agar (Sigma, St. Louis, MO, USA). The agar-cell mixture was plated on top of a bottom layer consisting of $1 \%$ agar in complete medium. After 15 days, colony size was measured using an ocular micrometer and colonies larger than $0.1 \mathrm{~mm}$ in diameter were counted. The experiment was performed three times for each cell line.

5-Ethynyl-2'-deoxyuridine assay. Cells were transfected with miRNA mimics in 96-well plates. Forty-eight hours after transfection, 5-ethynyl-2'-deoxyuridine (EdU) $(100 \mu \mathrm{M})($ Cell Light EdU DNA imaging kit; Guangzhou RiboBio Co., Ltd., Guangzhou, China) was added, and the cells were cultured for an additional $2 \mathrm{~h}$. The cells were then stained according to the production manual (Guangzhou RiboBio Co., Ltd.). Images were captured and analyzed using fluorescence microscopy (Nikon Eclipse 80i; Nikon, Tokyo, Japan). EdU-positive cells were determined with the formula: (EdU-treated cells/DAPI stained cells) $\mathrm{x} 100 \%$.

Flow cytometry. The cells were fixed in $70 \%$ ice-cold ethanol for $48 \mathrm{~h}$ at $4^{\circ} \mathrm{C}$ and stained by incubation with PBS containing $10 \mu \mathrm{g} / \mathrm{ml}$ propidium iodide and $0.5 \mathrm{mg} / \mathrm{ml} \mathrm{RNaseA}$ for $15 \mathrm{~min}$ at $37^{\circ} \mathrm{C}$. The cells were analyzed for the DNA content of labeled cells by FACSCaliber cytometry (BD Biosciences, Franklin Lakes, NJ, USA). Each experiment was conducted in triplicate.

Dual-luciferase assay. The luciferase reporter constructs pEZX-MT01-WIF1 3'-UTR WT and miRNA target clone control vector for pEZX-MT01 were purchased from GeneCopoeia, Inc. (HmiT001390-MT01 and CmiT000001MT01). Mutant reporter plasmids were obtained from this plasmid using a KOD-Plus Mutagenesis kit (SMK-101; Toyobo Co., Ltd., Life Science Department, Osaka, Japan). Luciferase assays were conducted using 293T cells plated in a 24-well plate (NEST Biotechnology). Transfections were performed using Lipofectamine $^{\mathrm{TM}} 2000$ (11668-019; Invitrogen) in Opti-MEM serum-free media (cat. no. 10742; Gibco). Luciferase and Renilla signals were measured $48 \mathrm{~h}$ after transfection using the Dual-Luciferase Reporter Assay kit (Promega Corp., Madison, WI, USA) according to the manufacturer's instructions. Three 

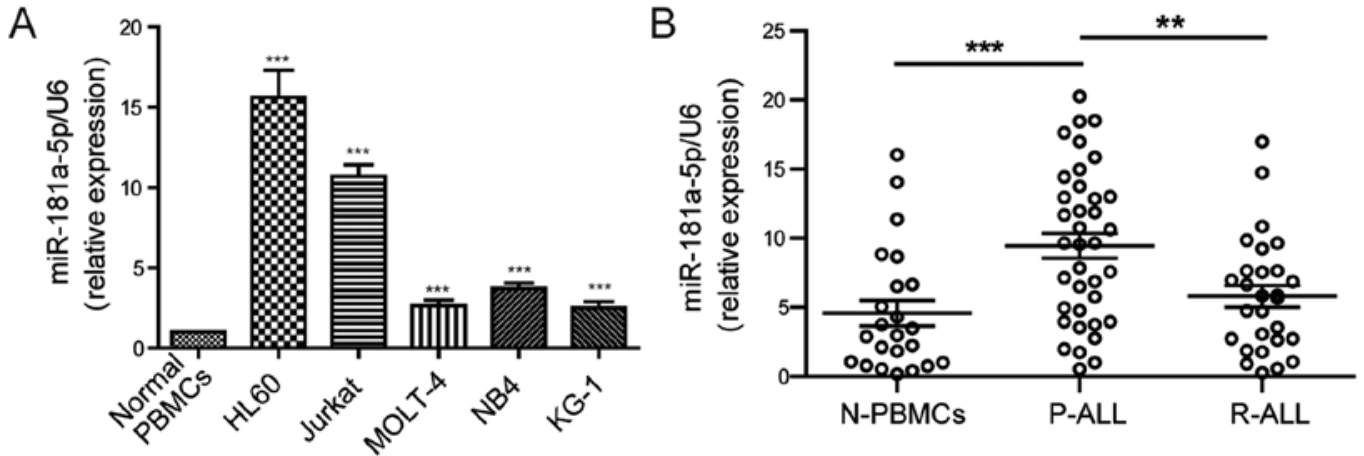

Figure 1. miR-181a-5p is overexpression in leukemia cell lines and primary acute lymphoblastic leukemia (ALL) patients. (A) Real-time PCR analysis of miR-181a-5p expression in five leukemia cell lines (HL60, MOLT-4, JURKAT, NB4 and KG-1), compared with the normal peripheral blood mononuclear cells (PBMCs) from healthy voluntary individuals. (B) miR-181a-5p expression detected by qRT-PCR in clinical samples. N-PBMC, normal PBMCs; P-ALL and R-ALL, primary or complete remission ALL patient' bone marrow samples. Values represent mean $\pm \mathrm{SD} ; \mathrm{n}=3,{ }^{* *} \mathrm{P}<0.01,{ }^{* * *} \mathrm{P}<0.001$.

independent experiments were performed, and the data are presented as the mean \pm SD.

Western blot assay. Cell lysate was prepared using RIPA buffer with protease inhibitors and quantified using the BCA protein assay (BioTek China, Beijing, China). The Nuclear Protein Extraction kit (BSP009; Sangon Biotech Co., Ltd., Shanghai, China) was used for extracting nuclear proteina of the ALL cells. Protein $(20 \mu \mathrm{g})$ was loaded onto a $10 \%$ SDS-PAGE gel that was then transferred onto a PVDF membrane and incubated with anti-WIF1 (1:1,000, ab2064; Cell Signaling Technology, Inc., Danvers, MA, USA), anti- $C-m y c$ (1:500, SC-40), antiE2F1 (1:1,000, SC-22820), anti-CDK4 (1:1,000, SC-260), anti-CCND1 (1:500, SC-753) and anti-P21 (1:1,000, SC-397) (all from Santa Cruz Biotechnology, Inc.) at $4^{\circ} \mathrm{C}$ overnight in blocker (3\% non-fat dry milk/BSA in TTBS) followed by incubation with HRP-conjugated secondary anti-mouse antibody (1:2,000, ZB2305; ZSGB-BIO, Beijing, China). Protein was normalized with GAPDH (1:5,000, no. P30008; Abmart, Shanghai, China) and histone-H3 (1:2,000, 17168-1-AP; ProteinTech Group, Inc., Chicago, IL, USA).

Statistical analysis. All statistical analyses were performed using the SPSS 13.0 Statistical Software Package (SPSS, Inc., Chicago, IL, USA). Two-tailed Student's t-test was used to determine the differences between groups for in vitro and in vivo analyses. The differences were considered to be statistically significant at a P-value $<0.05$. All data are presented as mean \pm SD or SEM unless otherwise noted.

\section{Results}

miR-181a-5p is highly expressed in various leukemia cell lines and primary $A L L$. RT-qPCR analyses initially showed that the expression of miR-181a-5p was markedly upregulated in 5 cell lines (HL60, MOLT-4, JURKAT, NB4 and KG-1) as compared with that in the normal PBMCs from healthy voluntary individuals (Fig. 1A). To validate the clinical relevance of miR-181a-5p to ALL patients, we further examined the miR-181a-5p expression level in 37 primary ALL samples and 28 ALL-complete remission samples compared with 23 PBMC samples. As shown in Fig. 1B, miR-181a-5p was highly expressed in the primary ALL patient samples. Notably, the expression level of miR-181a-5p was significantly reduced in the ALL-complete remission patient samples compared with primary ALL patients. These data suggest that miR-181a-5p may play an important role in the pathogenesis of ALL, and is associated with prognosis and may be valuable in the evaluation of therapeutic effects.

miR-181a-5p promotes ALL cell growth and proliferation in vitro. To explore the role of miR-181a-5p in the development and progression of ALL, we next examined its role in cellular proliferation. MOLT-4 cells with lower miR-181a-5p expression were used in the gain-of-function studies, whereas Jurkat cells with higher miR-181a-5p expression (Fig. 1A) were applied in the loss-of-function analyses. MTT and soft agar colony formation assays were conducted in the MOLT- 4 cells transfected with the miR-181a-5p mimic and in Jurkat cells transfected with the miR-181a-5p inhibitor. The miR-181a-5p mimic increased cell growth and anchorage-independent growth ability of the MOLT-4 cells (Fig. 2A and B), while the miR-181a-5p inhibitor markedly reduced cell growth and anchorage-independent growth ability of the Jurkat cells (Fig. 3A and B).

Using flow cytometric analysis, we further found that MOLT-4 cells transfected with the miR-181a-5p mimic exhibited a significantly reduced cell proportion in the $\mathrm{G} 1$ phase by $9.22 \pm 0.34 \%(\mathrm{P}<0.001)$ and increased proliferation $(\mathrm{S} / \mathrm{G} 2 / \mathrm{M}$ cell proportion) by $9.18 \pm 0.30 \%(\mathrm{P}<0.001)$ (Fig. $2 \mathrm{C})$, whereas the miR-181a-5p inhibitor increased the percentage of cells in the $\mathrm{G} 1$ phase by $9.02 \pm 0.83 \%(\mathrm{P}<0.001)$ and reduced the proliferation of Jurkat cells (S/G2/M cell proportion) by $9.08 \pm 0.47 \%$ $(\mathrm{P}<0.001)$ (Fig. 3C). Consistently, EdU incorporation assay showed that the percentage of cells in $\mathrm{S}$ phase was significantly increased in the miR-181a-5p-overexpressing MOLT-4 cells compared with the miR-control MOLT-4 cells (Fig. 2D), but the miR-181a-5p inhibitor reduced the percentage of Jurkat cells in the $\mathrm{S}$ phase (Fig. 3D). These results suggest that miR181a-5p modulates ALL cell proliferation through regulation of $\mathrm{G} 1 / \mathrm{S}$ transition.

WIF1 is a major target gene of miR-181a-5p in ALL cells. WIF1 is a Wnt antagonist that inhibits Wnt signaling by direct 


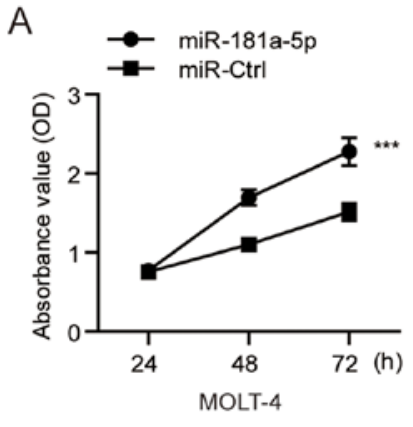

D
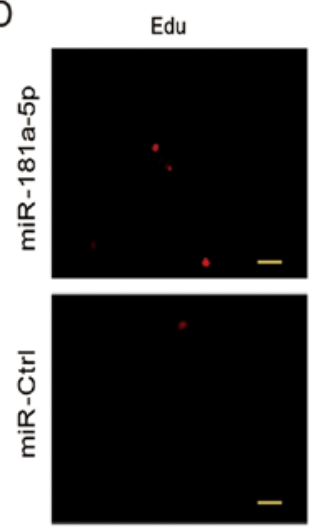

B

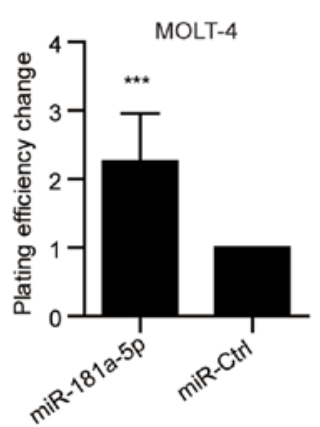

DAPI
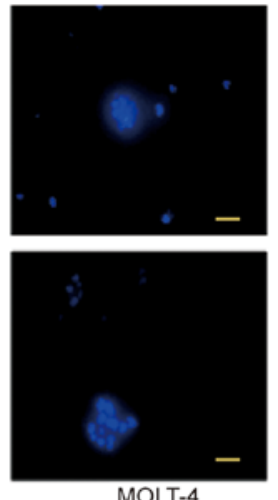
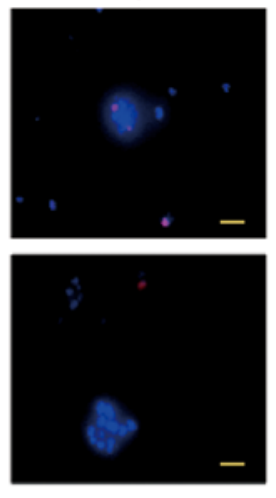

C
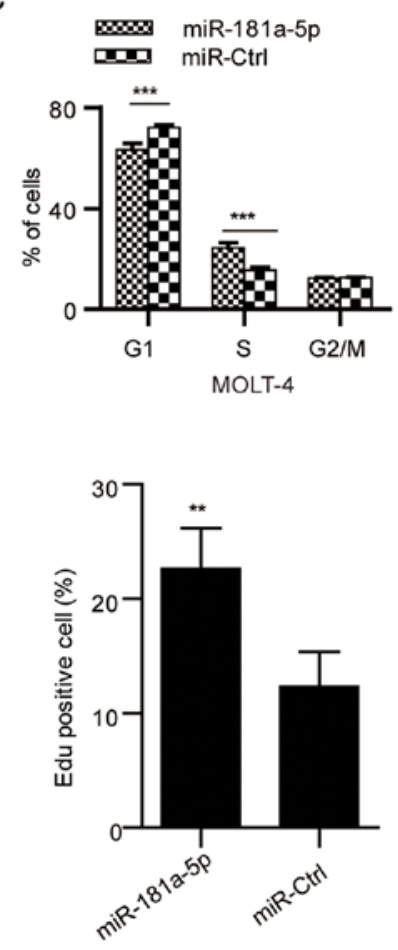

Figure 2. miR-181a-5p promotes acute lymphoblastic leukemia (ALL) cell growth and proliferation. (A) Effect of miR-181a-5p mimics on the proliferation of MOLT-4 as detected by MTT assay in triplicate. (B) Quantification of colony formation results of MOLT-4 cells in complete medium consisting of $1 \%$ agar (C) The cell cycle distribution was detected in the MOLT-4 cells following transfection with miR-181a-5p mimics (miR-181a-5p) or negative control (miR-Ctrl) by FACSCaliber cytometry. Data are presented as mean \pm SEM for three independent experiments. (D) Representative micrographs (left, 100x magnification; scale bar, $20 \mu \mathrm{m}$ ) and quantification of EdU-positive MOLT-4 cells (right) following transfection with miR-181a-5p mimics (miR-181a-5p) or negative control (miR-Ctrl). Values represent mean $\pm \mathrm{SEM} ;{ }^{* *} \mathrm{P}<0.01,{ }^{* * *} \mathrm{P}<0.001$.

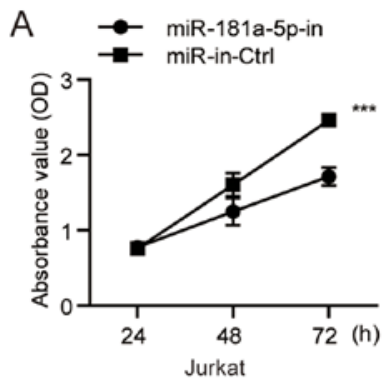

B

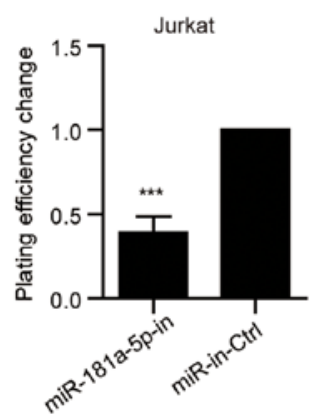

D
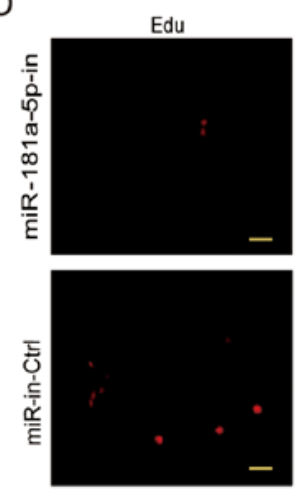
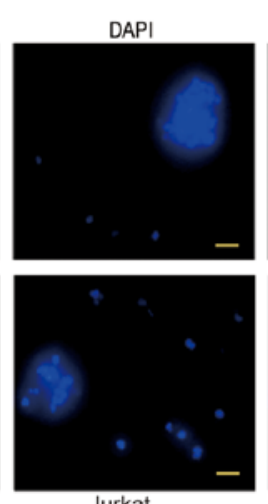
A

hsa-miR-181a-5p

position 51

target 5'

$\begin{array}{lcc} & \text { ACC } & \text { CA GUGUUGAAUGUU } \\ \text { miRNA 3' UGAG } & \text { CU } & \text { GU CGCAACUUAC AA }\end{array}$
B

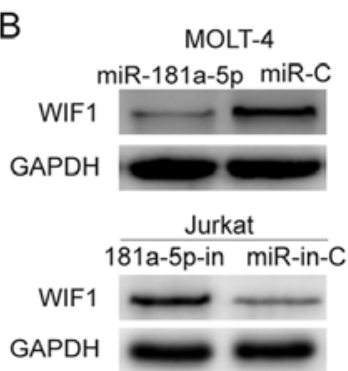

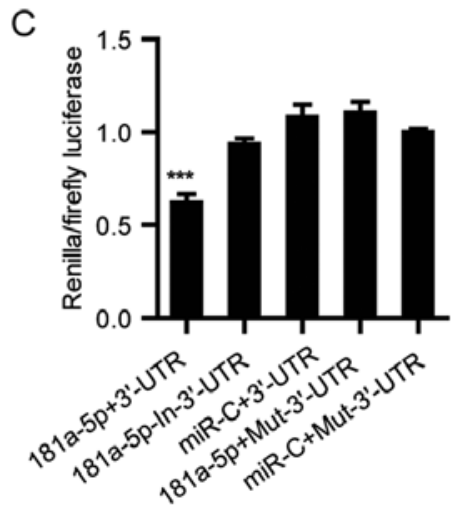

Figure 4. miR-181a-5p suppresses Wnt inhibitory factor-1 (WIF1) expression by directly targeting the WIF1 3'-UTR. (A) RNA hybrid prediction of the binding sites of miR-181a-5p in the 3'-UTR of WIF1. (B) WIF1 protein expression was detected by western blot analysis in the MOLT-4 and Jurkat cells transfected with the miR-181a-5p mimic and inhibitor, respectively. (C) Luciferase activities of dual-luciferase reporter carrying a complete wild-type or mutant of WIF1 $3^{\prime}$-UTR were assessed in the indicated cells in triplicate. Values represent mean $\pm \mathrm{SEM},{ }^{* * *} \mathrm{P}<0.001$.

Table I. Predicted microRNA according to mRNA selected regions (minimum seed length, 7; $\mathrm{P}<0.001$ ).

\begin{tabular}{|c|c|c|c|c|c|c|c|}
\hline microRNA & Gene & RefseqID & Seed length & Start & Seed sequence & End & P-value \\
\hline hsa-miR-181a & CPOX & NM_000097 & 11 & 2596 & AACAUUCAACG & 2586 & 0.0003 \\
\hline hsa-miR-181a & CHST9 & NM_031422 & 10 & 3110 & AACAUUCAAC & 3101 & 0.0006 \\
\hline hsa-miR-181a & HMGB2 & NM_002129 & 10 & 1055 & AACAUUCAAC & 1046 & 0.0007 \\
\hline hsa-miR-181a & IL2 & NM_000586 & 10 & 739 & AACAUUCAAC & 730 & 0.0003 \\
\hline hsa-miR-181a & SYNE1 & NM_182961 & 10 & 27647 & AACAUUCAAC & 27638 & 0.0007 \\
\hline hsa-miR-181a & WIF1 & NM_007191 & 10 & 1357 & AACAUUCAAC & 1348 & 0.0007 \\
\hline hsa-miR-181a & C12orf56 & NM_001099676 & 9 & 1529 & AACAUUCAA & 1521 & 0.0009 \\
\hline hsa-miR-181a & DNAJC3 & NM_006260 & 9 & 5051 & ACAUUCAAC & 5043 & 0.0000 \\
\hline hsa-miR-181a & ELAVL4 & NM_021952 & 9 & 1664 & AACAUUCAA & 1656 & 0.0009 \\
\hline hsa-miR-181a & HIGD2A & NM_138820 & 9 & 548 & AACAUUCAA & 540 & 0.0010 \\
\hline hsa-miR-181a & PIH1D2 & NM_001082619 & 9 & 1095 & AACAUUCAA & 1087 & 0.0007 \\
\hline hsa-miR-181a & SFT2D1 & NM_145169 & 9 & 546 & AACAUUCAA & 538 & 0.0008 \\
\hline hsa-miR-181a & CENPI & NM_006733 & 8 & 2550 & AACAUUCA & 2543 & 0.0005 \\
\hline hsa-miR-181a & KRTCAP2 & NM_173852 & 8 & 526 & AACAUUCA & 519 & 0.0009 \\
\hline hsa-miR-181a & NCR2 & NM_004828 & 8 & 940 & AACAUUCA & 933 & 0.0004 \\
\hline hsa-miR-181a & WDR79 & NM_018081 & 8 & 1836 & ACAUUCAA & 1829 & 0.0002 \\
\hline
\end{tabular}

WIF1, Wnt inhibitory factor-1.

binding to Wnt molecules. Using miRwalk, a publicly available algorithm, we found that WIF1 is theoretically the target gene of miR-181a-5p (Fig. 4A and Table I). To validate whether WIF1 is a direct target of miR-181a-5p, a wild-type or mutant 3'-UTR fragment of WIF1 was cloned downstream of the firefly luciferase gene. Dual-luciferase reporter assays revealed that miR-181a-5p significantly attenuated the activity of firefly luciferase with the wild-type 3'-UTR of WIF1, whereas this effect was abolished when the predicted 3'-UTR-binding site was mutated (Fig. 4B). Subsequent western blot analysis confirmed that miR-181a-5p attenuated the expression of cellular WIF1 in MOLT-4 cells and silencing of miR-181a-5p increased the level of cellular endogenous WIF1 protein in the Jurkat cells (Fig. 4C). These data suggest that miR-181a-5p may inhibit the expression of WIF1 by directly binding to its 3'-UTR.
WIF1 suppression is required for miR-181a-5p-induced ALL cell proliferation. To further confirm the role of WIF1 suppression in miR-181a-5p-induced ALL cell proliferation, we knocked down WIF1 expression in ALL cells (MOLT-4) using specific siRNA (Fig. 5E) and then observed the alteration of cell proliferation. Similar to miR-181a-5p, WIF1 siRNA enabled an obviously reduced WIF1 expression followed by increased ALL cell growth and proliferation (Fig. 5A-E). In addition, following treatment of Jurkat cells with the miR181a-5p inhibitor, significantly increased WIF1 expression and restricted cell proliferation were observed. Furthermore, we transfected WIF1-siRNA into the miR-181a-5p inhibitor-treated cells and observed that G1/S phase cell cycle arrest due to miR-181a-5p inhibition was abrogated (Fig. 5F and G). Taken together, these data suggest that WIF1 suppression is required for miR-181a-5p-induced ALL cell proliferation. 
A

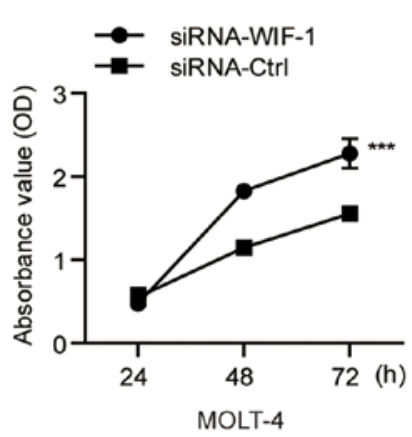

B
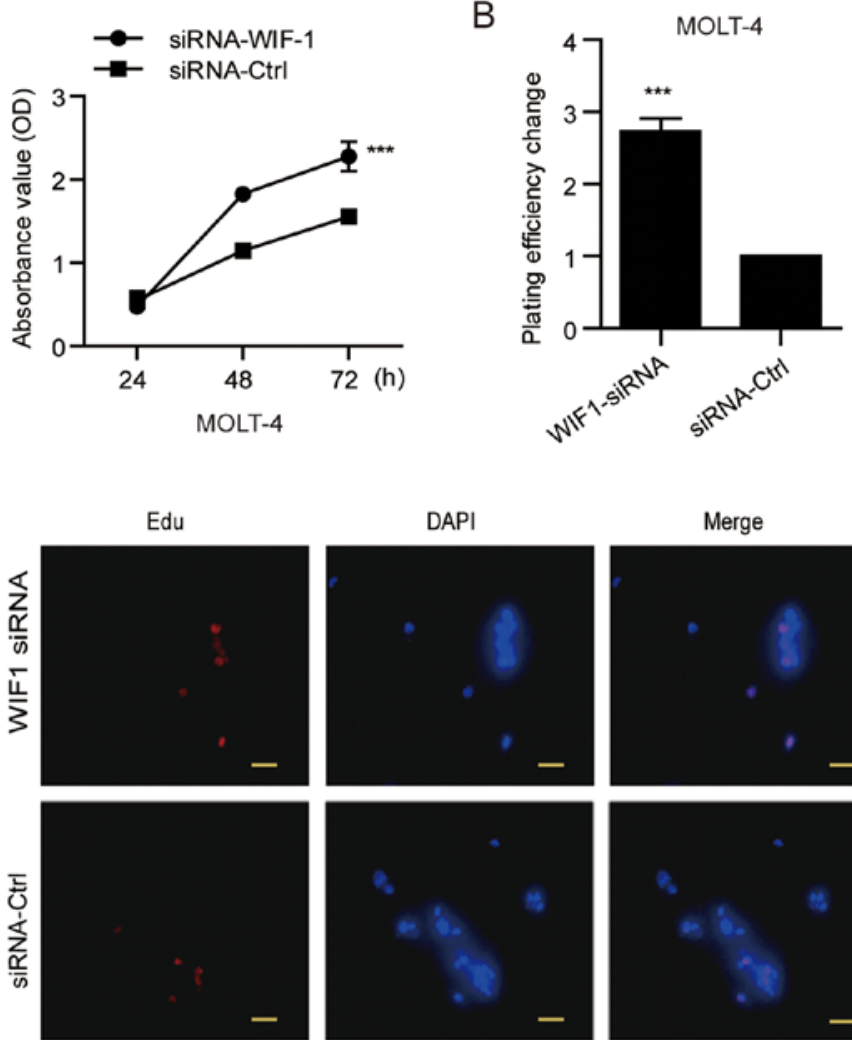

DAPI
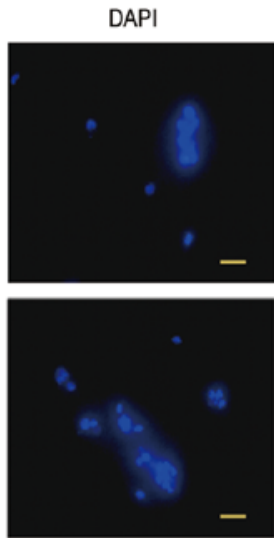

MOLT-4
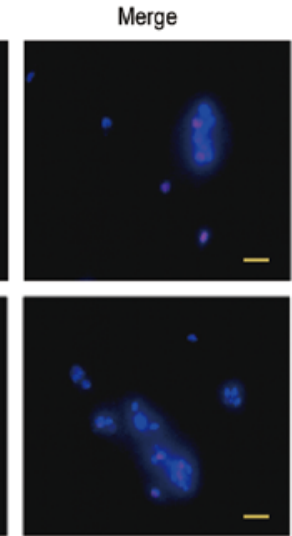
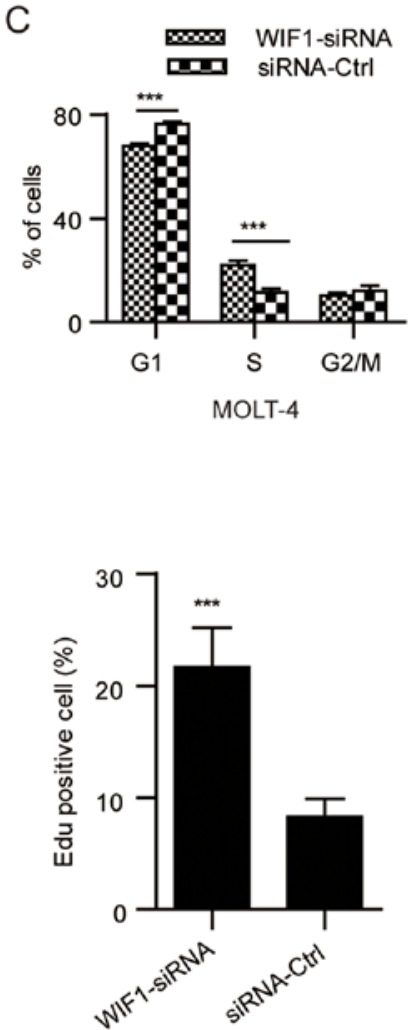

$E$

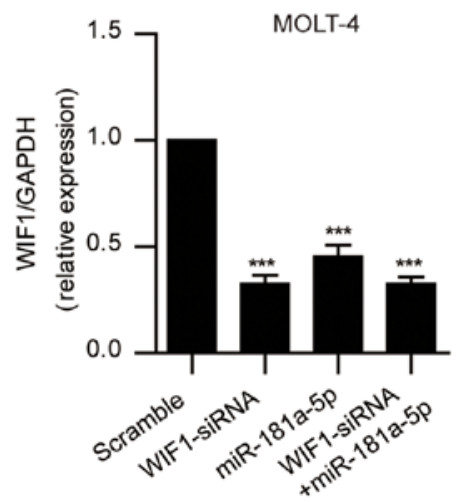

$\mathrm{F}$

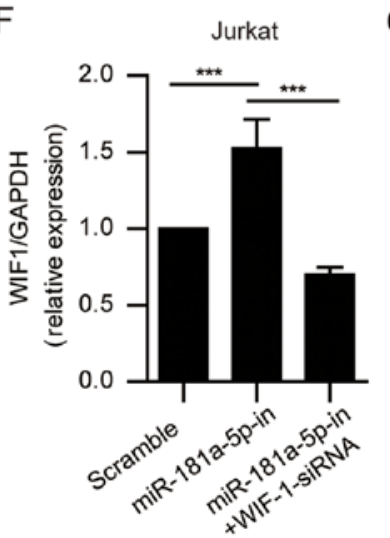

G

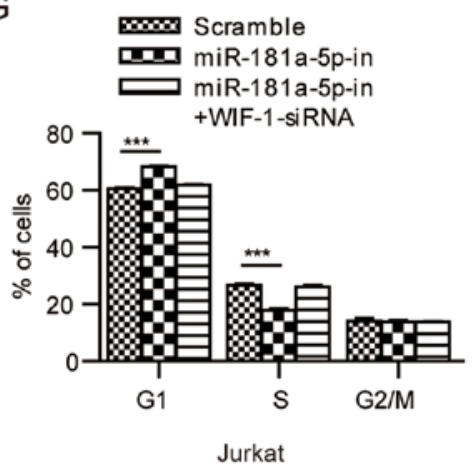

Figure 5. Wnt inhibitory factor-1 (WIF1) downregulation is required for miR-181a-5p-induced proliferation of ALL cells. (A) Effect of small interfering RNA against WIF1 (siRNA-WIF1 or WIF-1-siRNA) on MOLT-4 cell proliferation as detected by MTT assay in triplicate. (B) Quantification of colony formation results of MOLT-4 cells treated with small interfering RNA against WIF1 and negative control in complete medium consisting $1 \%$ agar. (C) The cell cycle distribution was detected in the indicated MOLT-4 cells by FACSCaliber cytometry. (D) Representative micrographs (left, 100x magnification; scale bar, $20 \mu \mathrm{m}$ ) and quantification of EdU-incorporating MOLT-4 cells (right) following transfection with small interfering RNA against WIF1 and negative control. (E and F) Real-time PCR analysis for the relative expression of WIF1 in the indicated cells. (G) The cell cycle distribution was detected in the indicated Jurkat cells by FACSCaliber cytometry. Data are presented as mean \pm SEM for three independent experiments, ${ }^{* * *} \mathrm{P}<0.001$.

miR-181a-5p activates Wnt/ $\beta$-catenin signaling through suppression of WIFl. The cellular fractionation and western blotting showed that miR-181a-5p overexpression promoted nuclear accumulation of $\beta$-catenin, indicating that miR-181a-5p may activate the $\mathrm{Wnt} / \beta$-catenin pathway through promoting nuclear $\beta$-catenin accumulation (Fig. 6A). Accordingly, we investigated whether miR-181a-5p influences downsteam signaling of the $\mathrm{Wnt} / \beta$-catenin pathway. As expected, the expression levels of c-myc, E2F1, cyclin D1, and CDK4 were increased while p21 expression was decreased following over- expression of miR-181a-5p. Opposite results were found in the miR-181a-5p-inhibited ALL cells (Fig. 6B).

\section{Discussion}

The expression and functions of specific miRNAs generally differ distinctly depending on the cancer cell type. In the present study, we observed that miR-181a-5p expression was highly expressed in several leukemia cell lines compared with that in normal PBMCs. Notably, we found that miR-181a-5p 
A

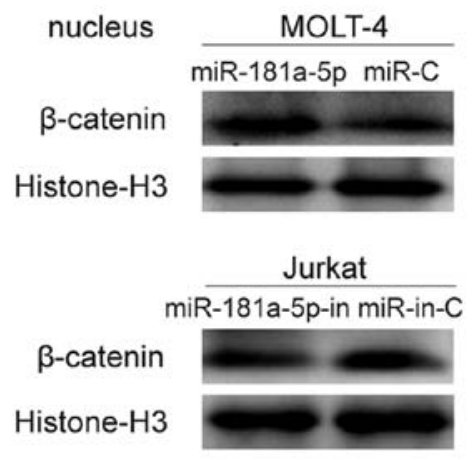

B

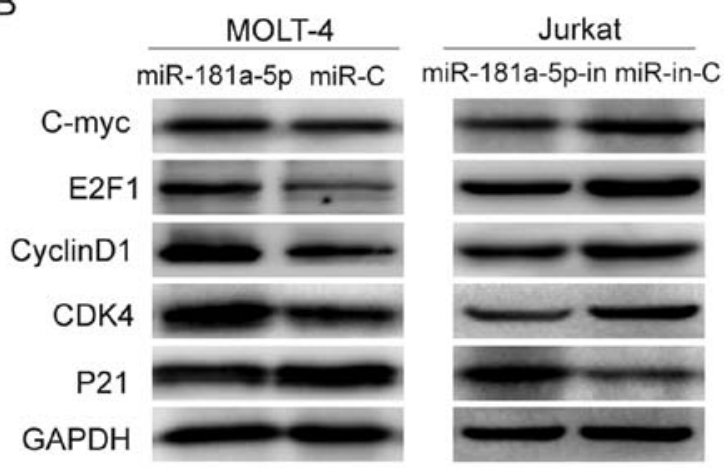

Figure 6. miR-181a-5p activates the Wnt/ $\beta$-catenin pathway. (A) Western blot analysis of $\beta$-catenin expression in nucleus of MOLT-4 and Jurkat cells transfected with miR-181a-5p mimic (miR-181a-5p) and inhibitor (miR-181a-5p-in) respectively. (B) Western blot analyses of $c-m y c, E 2 F 1, c y c l i n$ D1, CDK4 and p21 in the indicated cell groups.

was downregulated in ALL-complete remission samples compared with that in primary ALL samples, indicating that miR-181a-5p may be a potential biomarker for the evaluation of clinical efficacy and prognosis of ALL. This is consistent with a previous study that increased expression of miR-181a is associated with a poor outcome of ALL (39).

miR-181a can serve as an oncomiR or tumor suppressor, also implicating its complexity and muti-functions in the regulation of its target genes or signaling pathways in cancer. In the present study, we found that the ectopic expression of miR-181a enhanced ALL cell proliferation and silencing of miR-181a expression displayed the opposite effect, suggesting that abnormal miR-181a expression may contribute to the pathogenesis of ALL. Furthermore, we used a bioinformatics approach to predict the target genes of hsa-miR-181a-5p. Among the potential candidates, WIF1 attracted our attention since it is a classic tumor-suppressor gene involved in the cell proliferation in various cancers (40-42). Importantly, $\mathrm{Wnt} / \beta$-catenin signaling is frequently activated in $\operatorname{ALL}(43,44)$, but its precise mechanism of action is not well understood. WIF1 has proved to act as a secreted Wnt inhibitor that binds directly to Wnt, and thus constraining the binding of Wnt ligands to the Frizzled receptor (45). Thus, the downregulation of WIF1 contributes to activation of the Wnt pathway resulting in carcinogenesis through dysregulation of cell proliferation and differentiation. Luciferase reporter assays and western blotting further confirmed that miR-181a-5p directly suppressed WIF1 expression via directly targeting the 3'-UTR of WIF1. Although it has been reported that WIF1 is a target gene of miR-181a in colorectal cancer (46), our study suggests that WIF1 is a major target of miR-181a-5p and inactivation induced by miR-181a-5p may play an important role in promoting the carcinogenesis of ALL.

$\beta$-catenin is a major cellular effector of Wnt signaling. It is normally targeted by a complex of axin, APC, glycogen synthase kinase- $3 \beta$ and casein kinase $1 \alpha$ for proteasomemediated degradation following phosphorylation and ubiquitination $(47,48)$. $\beta$-catenin accumulation and nuclear translocation to regulate genes are important for cell proliferation. Notably, we observed that high expression of miR-181a-5p led to increased nuclear accumulation of $\beta$-catenin, indicating that $\mathrm{miR}-181 \mathrm{a}-5 \mathrm{p}$ can activate $\mathrm{Wnt} / \beta$-catenin signaling in
ALL. Subsequently, we detected the downstream targets of activated Wnt pathway signaling, confirming the activation of Wnt/ $\beta$-catenin signaling in the presence of WIF1 inactivation via miR-181a-5p in ALL. Therefore, in vivo antagomir-based strategies may be researched for further understanding the basic and translational significance of miR-181a-5p-Wnt/ $\beta$ catenin signaling in ALL.

In conclusion, we demonstrated that WIF1 is a major target of miR-181a-5p in ALL and downregulation induced by miR-181a-5p activated $\mathrm{Wnt} / \beta$-catenin signaling, promoting the proliferation of ALL. These findings uncover a crucial molecular mechanism that maintains the constitutive activation of the $\mathrm{Wnt} / \beta$-catenin pathway and may prove to be clinically useful for developing a new curative and effective biomarker and therapeutic target for ALL.

\section{Acknowledgements}

This study was jointly funded by grants from the National Natural Science Foundation of China (no. 81502335) and Natural Science Foundation of Guangdong Province (no. S2014A030310028).

\section{References}

1. Pui $\mathrm{CH}$ and Evans WE: Treatment of acute lymphoblastic leukemia. N Engl J Med 354: 166-178, 2006.

2. Flotho C, Coustan-Smith E, Pei D, Cheng C, Song G, Pui CH, Downing JR and Campana D: A set of genes that regulate cell proliferation predicts treatment outcome in childhood acute lymphoblastic leukemia. Blood 110: 1271-1277, 2007.

3. Jabeen K, Ashraf MS, Iftikhar S and Belgaumi AF: The impact of socioeconomic factors on the outcome of childhood acute lymphoblastic leukemia (ALL) treatment in a low/middle income country (LMIC). J Pediatr Hematol Oncol 38: 587-596, 2016.

4. Dinmohamed AG, Szabó A, van der Mark M, Visser O, Sonneveld P, Cornelissen JJ, Jongen-Lavrencic $M$ and Rijneveld AW: Improved survival in adult patients with acute lymphoblastic leukemia in the Netherlands: A population-based study on treatment, trial participation and survival. Leukemia 30: 310-317, 2016.

5. Cruz-Rodriguez N, Combita AL, Enciso LJ, Quijano SM, Pinzon PL, Lozano OC, Castillo JS, Li L, Bareño J, Cardozo C, et al: High expression of ID family and IGJ genes signature as predictor of low induction treatment response and worst survival in adult Hispanic patients with B-acute lymphoblastic leukemia. J Exp Clin Cancer Res 35: 64, 2016. 
6. van Es JH, Barker N and Clevers H: You Wnt some, you lose some: Oncogenes in the Wnt signaling pathway. Curr Opin Genet Dev 13: 28-33, 2003.

7. Lee HC, Kim M and Wands JR: Wnt/Frizzled signaling in hepatocellular carcinoma. Front Biosci 11: 1901-1915, 2006.

8. Kim M, Lee HC, Tsedensodnom O, Hartley R, Lim YS, Yu E, Merle P and Wands JR: Functional interaction between Wnt 3 and Frizzled-7 leads to activation of the Wnt/beta-catenin signaling pathway in hepatocellular carcinoma cells. J Hepatol 48: 780-791, 2008 .

9. Wei W, Chua MS, Grepper S and So SK: Soluble Frizzled-7 receptor inhibits Wnt signaling and sensitizes hepatocellular carcinoma cells towards doxorubicin. Mol Cancer 10: 16, 2011.

10. Reya T, Duncan AW, Ailles L, Domen J, Scherer DC, Willert K, Hintz L, Nusse R and Weissman IL: A role for Wnt signalling in self-renewal of haematopoietic stem cells. Nature 423: 409-414, 2003.

11. Weerkamp F, van Dongen JJ and Staal FJ: Notch and Wnt signaling in T-lymphocyte development and acute lymphoblastic leukemia. Leukemia 20: 1197-1205, 2006.

12. Khan NI and Bendall LJ: Role of WNT signaling in normal and malignant hematopoiesis. Histol Histopathol 21: 761-774, 2006.

13. Staal FJ and Clevers HC: WNT signalling and haematopoiesis: A WNT-WNT situation. Nat Rev Immunol 5: 21-30, 2005.

14. Døsen G, Tenstad E, Nygren MK, Stubberud H, Funderud S and Rian E: Wnt expression and canonical Wnt signaling in human bone marrow B lymphopoiesis. BMC Immunol 7: 13, 2006.

15. Staal FJ and Sen JM: The canonical Wnt signaling pathway plays an important role in lymphopoiesis and hematopoiesis. Eur J Immunol 38: 1788-1794, 2008 .

16. Timm A and Grosschedl R: Wnt signaling in lymphopoiesis Curr Top Microbiol Immunol 290: 225-252, 2005.

17. Batra S, Shi Y, Kuchenbecker KM, He B, Reguart N, Mikami I, You L, Xu Z, Lin YC, Clément G, et al: Wnt inhibitory factor-1, a Wnt antagonist, is silenced by promoter hypermethylation in malignant pleural mesothelioma. Biochem Biophys Res Commun 342: 1228-1232, 2006.

18. Liu TH, Raval A, Chen SS, Matkovic JJ, Byrd JC and Plass C: CpG island methylation and expression of the secreted frizzled-related protein gene family in chronic lymphocytic leukemia. Cancer Res 66: 653-658, 2006.

19. Yau TO, Chan CY, Chan KL, Lee MF, Wong CM, Fan ST and $\mathrm{Ng}$ IO: HDPR 1, a novel inhibitor of the WNT/beta-catenin signaling, is frequently downregulated in hepatocellular carcinoma: Involvement of methylation-mediated gene silencing. Oncogene 24: 1607-1614, 2005.

20. Roman-Gomez J, Jimenez-Velasco A, Agirre X, Castillejo JA, Navarro G, Barrios M, Andreu EJ, Prosper F, Heiniger A and Torres A: Transcriptional silencing of the Dickkopfs-3 (Dkk-3) gene by $\mathrm{CpG}$ hypermethylation in acute lymphoblastic leukaemia Br J Cancer 91: 707-713, 2004.

21. Suzuki H, Watkins DN, Jair KW, Schuebel KE, Markowitz SD, Chen WD, Pretlow TP, Yang B, Akiyama Y, Van Engeland M, et al: Epigenetic inactivation of SFRP genes allows constitutive WNT signaling in colorectal cancer. Nat Genet 36: 417-422, 2004.

22. Mazieres J, He B, You L, Xu Z, Lee AY, Mikami I, Reguart N, Rosell R, McCormick F and Jablons DM: Wnt inhibitory factor-1 is silenced by promoter hypermethylation in human lung cancer. Cancer Res 64: 4717-4720, 2004.

23. Bartel DP: MicroRNAs: Genomics, biogenesis, mechanism, and function. Cell 116: 281-297, 2004.

24. Bartel DP: MicroRNAs: Target recognition and regulatory functions. Cell 136: 215-233, 2009.

25. Mori F, Strano S and Blandino G: MicroRNA-181a/b: Novel biomarkers to stratify breast cancer patients for PARPi treatment Cell Cycle 12: 1823-1824, 2013.

26. Taylor MA, Sossey-Alaoui K, Thompson CL, Danielpour D and Schiemann WP: TGF- $\beta$ upregulates miR-181a expression to promote breast cancer metastasis. J Clin Invest 123: 150-163, 2013.

27. Bisso A, Faleschini M, Zampa F, Capaci V, De Santa J, Santarpia L, Piazza S, Cappelletti V, Daidone M, Agami R, et al: Oncogenic miR-181a/b affect the DNA damage response in aggressive breast cancer. Cell Cycle 12: 1679-1687, 2013.

28. Jianwei Z, Fan L, Xiancheng L, Enzhong B, Shuai L and Can L: MicroRNA 181a improves proliferation and invasion, suppresses apoptosis of osteosarcoma cell. Tumour Biol 34: $3331-3337,2013$
29. Nishimura J, Handa R, Yamamoto H, Tanaka F, Shibata K, Mimori K, Takemasa I, Mizushima T, Ikeda M, Sekimoto M, et al: microRNA-181a is associated with poor prognosis of colorectal cancer. Oncol Rep 28: 2221-2226, 2012.

30. Zhang X, Nie Y, Du Y, Cao J, Shen B and Li Y: MicroRNA-181a promotes gastric cancer by negatively regulating tumor suppressor KLF6. Tumour Biol 33: 1589-1597, 2012.

31. He Q, Zhou X, Li S, Jin Y, Chen Z, Chen D, Cai Y, Liu Z, Zhao T and Wang A: MicroRNA-181a suppresses salivary adenoid cystic carcinoma metastasis by targeting MAPK-Snai2 pathway. Biochim Biophys Acta 1830: 5258-5266, 2013.

32. Zhou JY, Ma WL, Fei J, Ding DP, Shi R, Jiang L and Zheng WL: Effects of microRNA miR-181a on gene expression profiles of K562 cells. Nan Fang Yi Ke Da Xue Xue Bao 26: 606-609, 2006 (In Chinese).

33. Li Y, Kuscu C, Banach A, Zhang Q, Pulkoski-Gross A, Kim D, Liu J, Roth E, Li E, Shroyer KR, et al: miR-181a-5p inhibits cancer cell migration and angiogenesis via downregulation of matrix metalloproteinase-14. Cancer Res 75: 2674-2685, 2015.

34. Shin KH, Bae SD, Hong HS, Kim RH, Kang MK and Park NH: miR-181a shows tumor suppressive effect against oral squamous cell carcinoma cells by downregulating K-ras. Biochem Biophys Res Commun 404: 896-902, 2011.

35. Debernardi S, Skoulakis S, Molloy G, Chaplin T, Dixon-McIver A and Young BD: MicroRNA miR-181a correlates with morphological sub-class of acute myeloid leukaemia and the expression of its target genes in global genome-wide analysis. Leukemia 21: 912-916, 2007.

36. Pons A, Nomdedeu B, Navarro A, Gaya A, Gel B, Diaz T, Valera S, Rozman M, Belkaid M, Montserrat E, et al: Hematopoiesis-related microRNA expression in myelodysplastic syndromes. Leuk Lymphoma 50: 1854-1859, 2009.

37. Pichiorri F, Suh SS, Ladetto M, Kuehl M, Palumbo T, Drandi D, Taccioli C, Zanesi N, Alder H, Hagan JP, et al: MicroRNAs regulate critical genes associated with multiple myeloma pathogenesis. Proc Natl Acad Sci USA 105: 12885-12890, 2008.

38. Zhu DX, Zhu W, Fang C, Fan L, Zou ZJ, Wang YH, Liu P, Hong M, Miao KR, Liu P, et al: miR-181a/b significantly enhances drug sensitivity in chronic lymphocytic leukemia cells via targeting multiple anti-apoptosis genes. Carcinogenesis 33: 1294-1301, 2012

39. Wang Y, Li Z, He C, Wang D, Yuan X, Chen J and Jin J: MicroRNAs expression signatures are associated with lineage and survival in acute leukemias. Blood Cells Mol Dis 44: 191-197, 2010.

40. Roperch JP, Incitti R, Forbin S, Bard F, Mansour H, Mesli F, Baumgaertner I, Brunetti $F$ and Sobhani I: Aberrant methylation of NPY, PENK, and WIF1 as a promising marker for blood-based diagnosis of colorectal cancer. BMC Cancer 13: 566, 2013.

41. Alvarez C, Tapia T, Cornejo V, Fernandez W, Muñoz A, Camus M, Alvarez M, Devoto L and Carvallo P: Silencing of tumor suppressor genes RASSF1A, SLIT2, and WIF1 by promoter hypermethylation in hereditary breast cancer. Mol Carcinog 52: 475-487, 2013.

42. Tang Y, Simoneau AR, Liao WX, Yi G, Hope C, Liu F, Li S, Xie J, Holcombe RF, Jurnak FA, et al: WIF1, a Wnt pathway inhibitor, regulates SKP2 and c-myc expression leading to G1 arrest and growth inhibition of human invasive urinary bladder cancer cells. Mol Cancer Ther 8: 458-468, 2009.

43. Nygren MK, Døsen-Dahl G, Stubberud H, Wälchli S, Munthe E and Rian E: beta-catenin is involved in N-cadherin-dependent adhesion, butnotincanonical Wnt signaling in E2A-PBX1-positive B acute lymphoblastic leukemia cells. Exp Hematol 37: 225-233, 2009.

44. Shao N, Zou J, Li J, Chen F, Dai J, Qu X, Sun X, Ma D and Ji C: Hyper-activation of WNT/ $\beta$-catenin signaling pathway mediates anti-tumor effects of histone deacetylase inhibitors in acute $\mathrm{T}$ lymphoblastic leukemia. Leuk Lymphoma 53: 1769-1778, 2012.

45. Hsieh JC, Kodjabachian L, Rebbert ML, Rattner A, Smallwood PM, Samos CH, Nusse R, Dawid IB and Nathans J: A new secreted protein that binds to Wnt proteins and inhibits their activities. Nature 398: 431-436, 1999.

46. Ji D, Chen Z, Li M, Zhan T, Yao Y, Zhang Z, Xi J, Yan L and Gu J: MicroRNA-181a promotes tumor growth and liver metastasis in colorectal cancer by targeting the tumor suppressor WIF-1. Mol Cancer 13: 86, 2014.

47. Willert K and Nusse R: Beta-catenin: A key mediator of Wnt signaling. Curr Opin Genet Dev 8: 95-102, 1998.

48. Clevers $\mathrm{H}$ and Nusse $\mathrm{R}$ : Wnt $/ \beta$-catenin signaling and disease. Cell 149: 1192-1205, 2012. 